\title{
Relire l'"Éducation sentimentale", textes réunis et présentés par Pierre GLAUDES et Eléonore REVERZY
}

\author{
Ida Merello
}

\section{OpenEdition}

\section{Journals}

\section{Edizione digitale}

URL: http://journals.openedition.org/studifrancesi/15690

DOI: 10.4000/studifrancesi. 15690

ISSN: 2421-5856

\section{Editore}

Rosenberg \& Sellier

\section{Edizione cartacea}

Data di pubblicazione: 1 décembre 2018

Paginazione: $519-521$

ISSN: 0039-2944

\section{Notizia bibliografica digitale}

Ida Merello, "Relire I"Éducation sentimentale", textes réunis et présentés par Pierre GLAUDEs et Eléonore REVERZY», Studi Francesi [Online], 186 (LXII | III) | 2018, online dal 01 janvier 2019, consultato il 06 janvier 2021. URL: http://journals.openedition.org/studifrancesi/15690 ; DOI: https://doi.org/10.4000/ studifrancesi. 15690

Questo documento è stato generato automaticamente il 6 janvier 2021.

\section{(†) $\odot$

Studi Francesi è distribuita con Licenza Creative Commons Attribuzione - Non commerciale - Non opere derivate 4.0 Internazionale. 


\title{
Relire l"Éducation sentimentale", textes réunis et présentés par Pierre GLAUDES et Eléonore REVERZY
}

\author{
Ida Merello
}

\section{NOTIZIA}

Relire l"Éducation sentimentale", textes réunis et présentés par Pierre GLAUDES et Eléonore REVERZY, Paris, Classiques Garnier 2017, 351 pp.

1 I curatori partono dalla considerazione di Flaubert dell'insuccesso dell'opera, per mostrare invece come l'accoglienza non fosse stata così negativa, e per rivederla in visione prospettica, in occasione dell'agrégation del 2018.

2 Yvan LECLERC, L"Éducation sentimentale" ou la "difficulté de trouver un bon titre», pp. 15-36. Il titolo si può leggere sotto il piano politico, come forma di educazione collettiva all'insegna della sentimentalità, ma Flaubert è convinto che la sentimentalità non si educa, e più radicalmente che la natura fa tutto, o quasi tutto. Ancora riflettendo sul titolo, e indagando nella corrispondenza, Leclerc ricorda che Flaubert, in una dedica dell'Éducation a Meilhac, aveva indicato come titolo migliore per l'opera Fruits secs; mentre in una lettera a George Sand riconosce che il titolo Forces perdues, già utilizzato da Du Camp, era il più adatto a indicare gli uomini della propria generazione, e quindi migliore di Éducation sentimentale.

3 Eric Le CALVEZ, Topographie et poétique du récit, pp. 37-50. Nel ricordare l'ostilità per le poetiche da parte di Flaubert, a vantaggio di una poetica intrinseca all'opera, l'A. mostra nell'E.S. la ricerca di un metodo che presieda alla struttura. La prima attenzione è allo spazio, sottomesso a un progetto realistico, anche per la necessità di rigore storico. Convinto che per far sognare occorre prima far immaginare, Flaubert costruisce un solido impianto realistico e storico, con una documentazione minuziosa, per poi orientare il lettore. In tal modo le descrizioni sono strettamente collegate alle 
emozioni, e anche la toponomastica diventa espressione di un luogo dell'anima, di un'emozione particolare.

4 Stéphanie DORD-CRousLÉ, Le personnage de Mme Arnoux, pp. 51-68. Il personaggio, frutto dell'influenza di molte donne, conosciute anche per via epistolare, non è dipinto con una personalità, ma come un vuoto, costantemente riempito da coloro che la guardano, ciascuno in maniera diversa. Al centro dell'articolo la sua identità borghese e il comportamento ipocrita legato ai costumi della buona borghesia. Madonna, santa, sfinge, Mme Arnoux è nascostamente madre, come starebbe a sottolineare il primo nome che le aveva attribuito Flaubert: Mme Moreau, ossia lo stesso di Frédéric. Ma l'immagine ha una costante degradazione oltre che fisica anche morale, in quanto legata al denaro, fin nella scena finale quando restituisce il debito, con un'analogia forte con il quadro di Rosanette che lei stessa scorge, nell'atto di presentare, nuda, un portamonete. Quando Flaubert parla di lei nelle sue lettere la descrive come un personaggio mediocre al pari di Frédéric, legata al suo destino piccolo borghese perfettamente immobile.

5 Borys LYON-CAEN, Intérieur avec vue. Frédéric est-il un personnage de roman?, pp. 69-86. Anche Frédéric appare per l'autore un personaggio cavo, vitale solo quando è messo in relazione con l'ambiente circostante, per cui funziona come occhio. L'abilità di Flaubert consiste nel far coincidere in maniera impercettibile l'occhio del narratore con il suo, che costituisce un fattore di coagulazione del testo. La sua interiorità è solo supposta. L'A. fa riferimento al flusso di coscienza, per mostrare la totale coincidenza con la voce del narratore. Solo quando ha una consistenza minima Frédéric diventa una persona e i suoi sogni sono un meccanismo per produrre finzioni.

6 François VANOOSThUYSE, La narration par «plans» dans l"Éducation sentimentale", pp.103-118. Secondo l'A. il cinema si è impadronito di tecniche flaubertiane: oltre all'uso del piano sequenza, forse introdotto da Stendhal, l'innescarsi della narrazione a partire dalle percezioni, la scoperta delle potenzialità emozionali della visione in movimento, l'interesse per i movimenti di luce negli spazi notturni e la scenarizzazione precisa degli spostamenti. L'A. scende poi nei dettagli dello stile, mostrando come l'E.S. funzioni bene come romanzo d'inchiesta: inchiesta che Frédéric può anche non concludere, ma il lettore sì. La scrittura per piani moltiplica le occasioni di approssimazioni, acuite dalla reticenza dello sguardo; al contrario ogni sequenza, isolando una situazione, offre suggerimenti.

7 Eléonore REVERZY, Le romanesque dans l'“Education sentimentale", pp. 103-118. Zola vede l'E.S. come espressione di una «platitude épique» e della «négation du romanesque dans l'intrigue»; lo stesso Flaubert a sua volta parla di personaggi medi, quindi ugualmente piatti. Se l'E.S. è diventata modello per il nouveau roman, lo si deve per l'A. proprio a questa forma di "deromanzizzazione", che nasconde ogni rapporto di causa e effetto per esibire invece le forme di casualità e capriccio, anche sotto il punto di vista grammaticale con "alors" e "ensuite". L'A mostra come le casualità siano in realtà molto preparate, in un contrasto tra piano sotterraneo e piano narrativo dove si manifesta l'ironia nei confronti del genere romanzesco e della vita.

8 Guy LARRouX, «Il parle bien, Frédéric Moreau», pp. 119-136. L’A. analizza i diversi livelli di conversazione dell'E.S.. Per prima cosa mostra che Frédéric Moreau non solo è timido e discreto, ma anche si imbroglia con le parole e non è padrone della situazione neppure a casa sua. Descrive quindi la conversazione come modo di dissimulazione, presente anche al di sotto della parola, come forma di comportamento che ad esempio acquisisce 
subito Mme Arnoux in presenza dei domestici. Tra i vari tipi di conversazione analizzati, prevalgono gli atti di linguaggio brevissimi. Solo nella soirée presso i Dambreuse il discorso di Frédéric si ricollega alla tradizione del codice romanzesco, e Frédéric parlando di Dussardier ha grande successo.

9 Gilles PHILIPPE, 1857-1869: un changement de style?, pp.137-151. Difficile rendere conto della complessità dello studio, che scende nei dettagli linguistici (per esempio l'uso, negli infiniti passati, della separazione di pas da ne, per evitare la serie dei "mots outils"), in modo da delineare in Flaubert un percorso che da Mme Bovary all'E.S. lo porta a uniformarsi a tendenze in atto.

Jeanne BEM, L“Éducation sentimentale": modernité et mobilité, pp. 153-172. L'A. accosta Mme Bovary e l'E.S., due romanzi di città che rispecchiano prima l'abitudine normanna di recarsi a Rouen, poi l'amore per Parigi. La città costituisce un polo di desiderio, e proprio la corsa del fiacre in Mme Bovary è la rappresentazione della città disincarnata e abitata dal desiderio. Flaubert parte dall'influenza di Balzac per arrivare a un'immagine personale, dove è forte il legame con l'acqua. L'A. trova punti di contatto con Baudelaire e Monet, ma anche con Perec e il cinema.

11 Christophe REFFAIT, Logique de l'argent et du récit dans l'Éducation sentimentale", pp.173-192. Se la descrizione dello scorrere del denaro è uno degli agenti di superficie della narrazione, anche la struttura appare dominata da questa logica, dal momento che, nell'esibizione dell'ineguaglianza sociale, tutto si svolge in base alla ricerca di contrastare i fallimenti e i crolli finanziari con altri capitali.

12 Nicolas Bourguinat, De Séville à Barcelone. L'Espagne dans l'“Éducation sentimentale", pp.173-210. L'E.S., grazie al realismo documentario, è un romanzo cosmopolita, che attira l'attenzione sulle diverse nazionalità; molte sono le allusioni alla Spagna: così la bellezza di Mme Arnoux è di tipo andaluso, inseguendo il gusto dell'epoca. Tuttavia l'A. nota che nessun personaggio è un rifugiato politico, tranne il «patriote de Barcelone», che appare nel capitolo della rivoluzione di febbraio. L'A. mostra che il discorso del patriota è un montaggio di una pagina di Alphonse Lucas che evocava un club democratico iberico. Ma il discorso proposto da Flaubert, con la sua qualità istrionica, distrugge la sognante rêverie ispanista del romanzo.

Gisèle SÉGINGER, L'histoire des sentiments et des émotions, pp.211-230. Aderendo alla sensibilità di Flaubert, l'A. vede come la sua attenzione storica non parta da teorie storiografiche, ma derivi dalle forme instabili dei discorsi e delle rappresentazioni, che alimentano quella che l'A. definisce la «force obscure» della Storia, ossia l'opinione. L'E.S. presenta un altro livello di storicità, non solo diverso dall'attenzione agli avvenimenti, ma anche ai costumi, basato su un livello infrastorico di motivazioni soggettive, inconsce o inconfessabili. È il momento dell'invidia democratica, con le conseguenze intellettuali ed estetiche dell'affermazione della mediocrità.

Jacques NEEFS, Le sentiment politique dans l'"Éducation sentimentale", pp. 231-244. In uno scambio di lettere con George Sand, Flaubert si lamenta della critica virulenta contro l'E.S., romanzo peraltro di successo. L'A. passa in rassegna la critica negativa. Inevitabile l'acrimonia di Barbey D'Aurevilly, che da sempre aveva fiutato in lui l'uomo antireligioso in tutti i suoi aspetti; ma anche Sarcey malignamente rimprovera l'eccesso di descrizioni. L'A. mette in evidenza invece la finezza di George Sand, che sa comprendere il valore e ribattere con buone argomentazioni alle accuse. Il senso 
dell'E.S. è politico, essendovi descritte la disfatta delle aspirazioni per la violenza del potere, e il concetto di libertà.

Pierre GLAUDES, Avatars du religieux dans l'“Éducation sentimentale", pp. 245-278. In un lavoro troppo complesso per lo spazio di una scheda, l'A. sottolinea gli elementi che Flaubert esibisce per mostrare la decristianizzazione della società dopo la rivoluzione del 1848: da un lato le pratiche religiose non più comprese, ma di cui si ripetono meccanicamente le parole, il bric à brac delle cappelle cimiteriali, che alludono ai simboli religiosi più disparati, come la chiesa dell'Alhambra, dall'altra il rigido zelo del fanatismo rivoluzionario, basato sugli stessi principi della religione, che mostra il passaggio di uno stesso atteggiamento da un campo all'altro. In mezzo, l'opportunismo che sembra rispondere perfettamente alla definizione di religion nel Dictionnaire des idées reçues: «nécessaire pour le peuple [...] cependant point trop n'en faut».

Fabrice wilhelm, Amitié et envie dans l"“Éducation sentimentale", pp. 281-296. L'invidia, ricorda l'A., può solo appartenere all'antagonista. Perciò nell'E.S. si esprime in tutta la gamma in Deslauriers, non in Frédéric, dominato da una fondamentale presunzione che gli fa regolare su questa tutti i rapporti umani. A parte l'eroe Dussardier, l'invidia si manifesta sia tra pari, sia tra inferiore e superiore, diventando per Flaubert un punto di osservazione delle dinamiche sociali oltre che di quelle individuali.

17 Juliette AzoUlaI, «Le bonheur peut y tenir»: conceptions du bonheur dans l'“Éducation sentimentale", pp. 297-314. L'A. riconosce la difficoltà di trovare una manifestazione di felicità all'interno dell'E.S., dove invece sono molteplici gli accenni al suo carattere illusorio. Eppure al contrario di Flaubert, il personaggio di Frédéric cede all'illusione di felicità con Rosanette e conosce le gioie della vanità, pur rinviando al miraggio la felicità assoluta. Lo stesso tipo di atteggiamento si ritrova in chiave politica, dove la realizzazione dell'utopia è rimandata al futuro. Anche se Flaubert confessa in una lettera a Louise Colet di non conoscere la felicità, non mancano nel romanzo i momenti di appagamento e una forma di iniziazione a una felicità "filosofica" superiore.

Jacques-David EBGUY, "Quel est le sens de tout cela?». L"Éducation sentimentale", roman du retrait, pp.315-336. Per l'A. la questione centrale dell'E.S. è la ricerca del senso. Il romanzo è fondato sulla sospensione dei personaggi di fronte all'azione, la riserva delle parole, un'estetica globale del non incatenamento, ma della giustapposizione di blocchi. In tal modo si creano vuoti, che permettono alle insorgenze improvvise di stagliarsi più nette, facendo risaltare le separazioni. Una simile costruzione, il cui senso finale è la creazione artistica, richiede un lettore attivo, che a sua volta deve scegliere e stabilire nessi. 